\title{
Effect of RNA integrity on uniquely mapped reads in RNA-Seq
}

Emily A Chen ${ }^{1 *}$, Tade Souaiaia ${ }^{2}$, Jennifer S Herstein ${ }^{2}$, Oleg V Evgrafov ${ }^{2}$, Valeria N Spitsyna ${ }^{2}$, Danea F Rebolini ${ }^{3}$ and James A Knowles ${ }^{2}$

\begin{abstract}
Background: We examined the performance of three RNA-Sequencing library preparation protocols as a function of RNA integrity, comparing gene expressions between heat-degraded samples to their high-quality counterparts. This work is invaluable given the difficulty of obtaining high-quality RNA from tissues, particularly those from individuals with disease phenotypes.
\end{abstract}

Results: With the integrity of total RNA being a critical parameter for RNA-Sequencing analysis, degraded RNA can heavily influence the results of gene expression profiles. We discovered that gene expression read results are influenced by RNA quality when a common library construction protocol is used. These results are based on one technical experiment from a pool of 4 neural progenitor cell lines.

Conclusions: The use of alternative protocols can allow samples with a wider range of RNA qualities to be used, facilitating the investigation of disease tissues.

Keywords: Gene expression, RNA-Sequencing, Low-quality RNA, Library construction protocol, Degradation

\section{Discussion}

\section{Background}

Adiconis et al. [1] examined the performance of five RNA-Seq sample preparation protocols when using RNA of low quality and/or quantity. This work is invaluable given the difficulty of obtaining high-quality RNA from tissues, particularly those from individuals with disease phenotypes. We have used a similar approach of evaluating the performance of RNA-Seq library preparation protocols, as a function of RNA integrity. We compared gene expression, as measured by RNA-Seq, of heat-degraded RNA samples to the expression profiles of the high-quality starting samples.

\section{Methods and results}

Specifically, 20 ug of high-quality total RNA (RIN 9.4; 2100 Bioanalyzer, Agilent Technologies Inc., Santa Clara, CA, USA) was constructed by pooling RNA extracted using a Direct-zol RNA MiniPrep kit (Zymo Research,

\footnotetext{
* Correspondence: emilyach@med.usc.edu

'Department of Genetic, Molecular, and Cellular Biology, Keck School of Medicine, Zilkha Neurogenetic Institute, University of Southern California, 1501 San Pablo Street, ZNI 401, MC 2821, Los Angeles, CA 90089-2821, USA Full list of author information is available at the end of the article
}

Irvine, CA, USA) from neural progenitor cell lines made from 4 individuals [2]. This pool was heat-degraded (60 minutes at $60^{\circ} \mathrm{C}$, followed by 6,20 and 30 mins at $90^{\circ} \mathrm{C}$ ) to RINs of 7.4, 5.3, and 4.5 [3]. RNA-Seq libraries were then made using three different protocols. 1) Poly-A RNA was purified from 1 ug of total RNA using oligo-dT beads, fragmented with divalent cations, made into cDNA and then sequencing libraries using the TruSeq RNA Sample Preparation kit v2 (RS-122-2001, Illumina Inc., San Diego, CA, USA). 2) Ribosomal RNA was removed from 1 ug of total RNA using the Ribo-Zero rRNA Removal kit (MRZH116, Epicentre Biotechnologies, Madison, WI, USA), and processed without the poly-A selection as per \#1.3) cDNA was made from 200 ng of total RNA using the Ovation RNASeq FFPE System (7150, NuGEN Technologies Inc., San Carlos, CA, USA), sheared to $300 \mathrm{bp}$ using a Covaris S2 (500003, Covaris Inc., Woburn, MA, USA), and followed by library construction using the TruSeq DNA Sample Preparation kit v2 (FC-121-2001).

Each DNA library was sequenced with 4.5-60 million $100 \mathrm{bp}$ single-end reads on an Illumina HiSeq2000. The

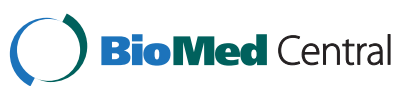

(c) 2014 Chen et al.; licensee BioMed Central Ltd. This is an Open Access article distributed under the terms of the Creative Commons Attribution License (http://creativecommons.org/licenses/by/4.0), which permits unrestricted use, distribution, and reproduction in any medium, provided the original work is properly credited. The Creative Commons Public Domain Dedication waiver (http://creativecommons.org/publicdomain/zero/1.0/) applies to the data made available in this article, unless otherwise stated. 


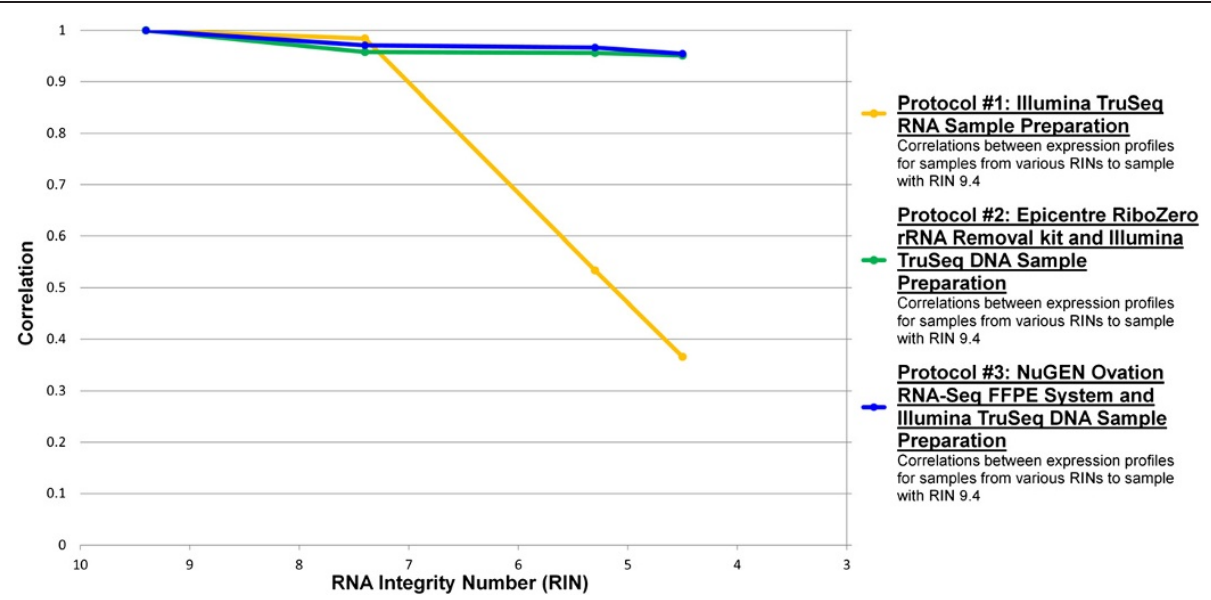

Figure 1 Effect of RNA integrity on gene expression correlations with untreated RNA using the same sample preparation protocol.

reads were uniquely mapped with three or fewer mismatches using PerM [4] to GENCODE v17. For each protocol, we calculated Pearson's pairwise correlation coefficients (denoted by the letter R) between the degraded and high-quality sample across the HUGO genes which contained at least one read alignment in either sample. $\mathrm{R}$ was calculated and depicted in Figure 1 by taking the $\log$ of (reads plus an offset of 1 ). All three protocols performed well at RIN 7.4 $(\mathrm{R}=0.958$ to 0.984 , s.e. $=0.001$ to 0.002$)$ (Figure 1, Table 1). However, as RNA quality decreased (RINs 5.3 and 4.5), protocol \#1 produced data with lower correlations of gene expression to the intact sample $(R=0.533$ and 0.366 , s.e. $=0.005)$. In contrast, both protocols \#2 and \#3 performed relatively well as RNA quality decreased $(\mathrm{R}=0.951$ to 0.967 , s.e. $=$ 0.002), with protocol \#3 performing slightly better. For each RIN quality, we calculated $\mathrm{R}$ between the reads from

Table $1 \mathrm{R}$ between degraded sample and intact sample for each protocol

\begin{tabular}{llll}
\hline Protocol & RIN & $\begin{array}{l}\text { R to RIN } \\
\mathbf{9 . 4} \text { sample }\end{array}$ & Total Reads \\
\hline NuGEN Ovation RNA-Seq FFPE & 9.4 & 1 & $37,959,903$ \\
System + Illumina TruSeq DNA & 7.4 & 0.971 & $51,131,950$ \\
Sample Preparation & 5.3 & 0.967 & $32,285,466$ \\
& 4.5 & 0.955 & $58,476,532$ \\
Epicentre RiboZero rRNA & 9.4 & 1 & $25,339,734$ \\
Removal Kit + Illumina TruSeq & 7.4 & 0.958 & $59,722,682$ \\
DNA Sample Preparation & 5.3 & 0.956 & $48,134,260$ \\
& 4.5 & 0.951 & $50,650,245$ \\
Illumina TruSeq RNA & 9.4 & 1 & $59,509,601$ \\
Sample Preparation & 7.4 & 0.984 & $35,262,055$ \\
& 5.3 & 0.533 & $20,939,261$ \\
& 4.5 & 0.366 & $4,531,318$ \\
\hline
\end{tabular}

each pair of protocols. The reads from the two best methods (Protocol \#2 and Protocol \#3) maintained high correlations regardless of decreased sample quality $(\mathrm{R}=$ 0.845 to 0.879 , s.e. $=0.003)$. For Protocol \#1, there was a drop in read correlation to both Protocol \#2 and Protocol \#3 as RIN decreased (Figure 2).

For confirmation of mapper accuracy, we mapped all of the samples using TopHat v1.4.0 [5] to GENCODE v17. The resulting BAM files were run through HTSeq v0.6.1 [6] to obtain uniquely mapped read counts. Essentially the same results were obtained as with PerM (data not shown). Additionally, to rule out any bias from differences in numbers of reads, we downsampled all of the samples to 4.5 million reads, and the results were essentially the same (data not shown).

\section{Conclusions}

It is likely that the poor performance of protocol \#1 at lower RINs can be explained by the poly-A selection step. As RNA integrity decreases, less full length polyA RNA is recovered, leading to a cDNA library that is increasingly 3 ' biased. This is supported by analysis of the $5^{\prime}$ to $3^{\prime}$ read distribution of each library. Those from protocols \#2 and \#3 are essentially unchanged at decreasing RIN, while the distribution for samples from protocol \#1 is severely 3' biased by RIN 4.5 (data not shown).

We recognize that our results are based on a single experiment using an RNA pool from 4 neural progenitor cell lines and are not broadly applicable. Hence, other investigators may want to use this method to determine the effect of RNA integrity on RNA-Seq from their tissue source of interest.

In summary, our data show that the results of RNA-Seq are influenced by RNA quality with a widely-used cDNA/ sequencing library construction protocol. However, this 


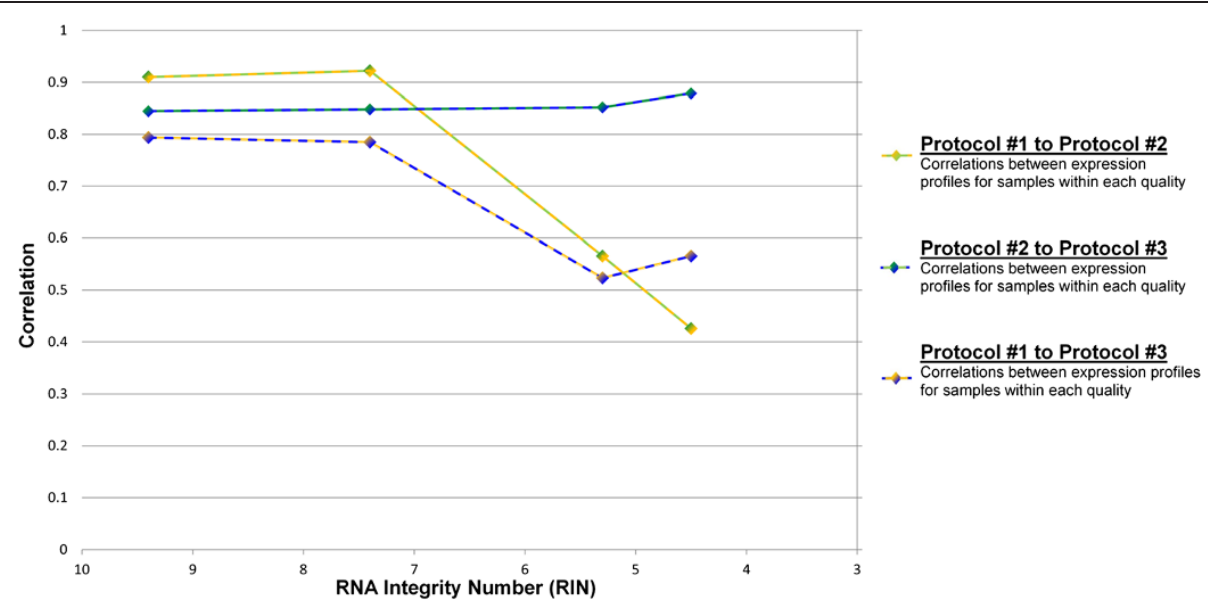

Figure 2 Effect of RNA integrity on gene expression correlations between different sample preparation protocols.

problem can be avoided with alternative protocols, allowing samples with a wider range of RNA qualities to be used, facilitating the investigation of disease tissues.

\section{Competing interests}

DR is currently employed at Illumina, Inc. JK received an honorarium for speaking at an Illumina, Inc. event.

\section{Authors' contributions}

EC carried out the sample preparation, data analysis and co-authored the manuscript. TS developed the mapping software. JH performed sequence alignment. OE advised on data analysis. VS extracted the RNA from the cell line. DR carried out the RNA sequencing. JK conceived the experiments, advised on data analysis and co-authored the manuscript. All authors read and approved the final manuscript.

\section{Acknowledgements}

This work was supported in part by research grants from the NIMH (MH090047 and MH086874), and the NHGRI (HG006531).

\section{Author details}

'Department of Genetic, Molecular, and Cellular Biology, Keck School of Medicine, Zilkha Neurogenetic Institute, University of Southern California, 1501 San Pablo Street, ZNI 401, MC 2821, Los Angeles, CA 90089-2821, USA. ${ }^{2}$ Department of Psychiatry and the Behavioral Sciences, Keck School of Medicine, Zilkha Neurogenetic Institute, University of Southern California, 1501 San Pablo Street, ZNI 401, MC 2821, Los Angeles, CA 90089-2821, USA. ${ }^{3}$ Illumina, Inc., 5200 Illumina Way, San Diego, CA 92122, USA.

Received: 20 March 2014 Accepted: 14 October 2014

Published: 23 October 2014

\section{References}

1. Adiconis X, Borges-Rivera D, Satija R, DeLuca DS, Busby MA, Berlin AM, Sivachenko A, Thompson DA, Wysoker A, Fennell T, Gnirke A, Pochet N, Regev A, Levin JZ: Comparative analysis of RNA sequencing methods for degraded or low-input samples. Nat Methods 2013, 10:623-629.

2. Evgrafov OV, Wrobel BB, Kang X, Simpson G, Malaspina D, Knowles JA: Olfactory neuroepithelium-derived neural progenitor cells as a model system for investigating the molecular mechanisms of neuropsychiatric disorders. Psychiatr Genet 2011, 21:217-228.

3. Opitz L, Salinas-Riester G, Grade M, Jung K, Jo P, Emons G, Ghadimi BM, Beissbarth T, Gaedcke J: Impact of RNA degradation on gene expression profiling. BMC Med Genomics 2010, 3:36.
4. Chen Y, Souaiaia T, Chen T: PerM: efficient mapping of short sequencing reads with periodic full sensitive spaced seeds. Bioinformatics 2009, 25:2514-2521

5. Trapnell C, Pachter L, Salzberg S: TopHat: discovering splice junctions with RNA-Seq. Bioinformatics 2009, 25:1105-1111.

6. Anders S, Pyl PT, Huber W: HTSeq - A Python framework to work with high-throughput sequencing data. BioRxiv preprint 2014, http://www.ncbi.nlm.nih.gov/pubmed/25260700.

doi:10.1186/1756-0500-7-753

Cite this article as: Chen et al: Effect of RNA integrity on uniquely mapped reads in RNA-Seq. BMC Research Notes 2014 7:753.

\section{Submit your next manuscript to BioMed Central and take full advantage of:}

- Convenient online submission

- Thorough peer review

- No space constraints or color figure charges

- Immediate publication on acceptance

- Inclusion in PubMed, CAS, Scopus and Google Scholar

- Research which is freely available for redistribution 